\title{
Analysis of Lymphocyte Profile in Lymph Nodes, Bronchoalveolar Lavage Fluid and Peripheral Blood in Patients With Stage II Sarcoidosis
}

\section{Song Mi}

Department of Pulmonary and Critical Care Medicine, Beijing Chao-Yang Hospital, Capital Medical University, Beijing

$\mathrm{Na}$ Cui

Department of Pulmonary and Critical Care Medicine, Beijing Chao-Yang Hospital, Capital Medical University, Beijing

\section{Xiaokai Feng}

Department of Pulmonary and Critical Care Medicine, Beijing Chao-Yang Hospital, Capital Medical University, Beijing

\section{Chunguo Jiang}

Department of Pulmonary and Critical Care Medicine, Beijing Chao-Yang Hospital, Capital Medical University, Beijing

\section{Jing Wang}

Department of Pulmonary and Critical Care Medicine, Beijing Chao-Yang Hospital, Capital Medical University, Beijing

\section{Liming Zhang ( $\nabla$ cyyyzlm@sina.com )}

Department of Pulmonary and Critical Care Medicine, Beijing Chao-Yang Hospital, Capital Medical University, Beijing

\section{Research Article}

Keywords: Sarcoidosis, Lymphocytes, Lymphocyte node, Bronchoalveolar lavage fluid, Endobronchial ultrasound-guided transbronchial needle aspiration(EBUS-TBNA)

Posted Date: November 8th, 2021

DOI: https://doi.org/10.21203/rs.3.rs-1034493/v1

License: (c) (1) This work is licensed under a Creative Commons Attribution 4.0 International License. Read Full License 


\section{Abstract}

Background: The lymphocyte profile(LP) in mediastinal lymph nodes involved by sarcoidosis has not been extensively studied and the results in the literature were mixed.

Methods: This was a prospective study of stage II sarcoidosis patients with intrathoracic lymphadenopathy underwent endobronchial ultrasound-guided transbronchial needle aspiration (EBUSTBNA) and bronchoalveolar lavage(BAL). LP in lymph node puncture fluid (LNPF),BAL fluid(BALF) and peripheral blood (PB) were evaluated using flow cytometry. The results of LP in PB,BALF and LNPF were compared. The relationship between respiratory symptoms and LP was preliminarily analyzed.

Results: In this study, 16 cases of stage II sarcoidosis were diagnosed, 11 with respiratory symptoms and 5 without. There was no correlation between LP in LNPF and BALF in all patients. Compared with the proportion of LP in BALF, in LNPF the lymphocytes and B cells increased, the T cells, CD4 , NK T cells and CD4/CD8 ratio decreased, and there was no difference between CD8 T cells and NK cells. Respiratory symptoms had no effect on LP in LNPF. T cells, CD4, CD8 T cells and CD4/CD8 ratio in BALF were correlated with respiratory symptoms, and there was significant difference between symptomatic group and asymptomatic group.

Conclusion: The LP in LNPF were significantly different from those in BALF and not correlated with BALF and not with respiratory symptoms, mainly reflect the involvement of lymph nodes, which may be more conducive to the diagnosis and differential diagnosis of sarcoidosis with lymph nodes involved. $T$ cells, CD4 T cells and CD4/CD8 ratio in BALF were positively correlated with respiratory symptoms, while CD8 in BALF and PB was negatively correlated with respiratory symptoms. These indexes may more accurately reflect the pulmonary involvement of sarcoidosis.

\section{Introduction}

Sarcoidosis is a multisystem inflammatory disorder of unknown aetiology. The lung is the most commonly affected organ, with heterogeneous involvement of the bronchovascular and subpleural parenchyma and intrathoracic lymph nodes. The diagnosis of sarcoidosis is not standardized and there are no universally accepted measures to determine if each diagnostic criterion has been satisfied; therefore, the diagnosis of sarcoidosis is never fully secure.

Analysis of bronchoalveolar lavage fluid (BALF) for total and differential counts of inflammatory cells and the CD4/CD8 ratio has been considered a useful supplement in the diagnostic workup of sarcoidosis ${ }^{[1]}$.In sarcoidosis, the characteristic findings in BALF are a T-cell-dominated lymphocytosis and a CD 4/CD8 ratio $\varangle 3.5^{[2]}$. However, the latter can be inverted and in elderly subjects also normally increased $^{[3]}$.

Mediastinal/hilar lymphadenopathy is one of the most important manifestations of sarcoidosis. Obtaining lymph node tissue samples for pathological examination is an important means for the 
diagnosis and differential diagnosis of sarcoidosis. The evaluation of intrathoracic lymphadenopathy can be performed using computed tomography (CT)-guided transthoracic needle aspiration, conventional transbronchial needle aspiration (TBNA), endobronchial ultrasound-guided TBNA (EBUS-TBNA), endoscopic ultrasound-guided fine-needle aspiration(EUS-FNA), and mediastinoscopy. Among these procedures, EBUS-TBNA is currently the preferred procedure as it is readily available, less invasive, and allows sampling of most intrathoracic lymph node stations under "direct" endosonographic vision ${ }^{[4]}$. Up to now, there are few investigations into LP in LNPF and into the relationship among PB,BALF and LNPF in patients with sarcoidosis. In this study, stage 2 sarcoidosis patients with mediastinal/hilar lymphadenopathy and lung tissue involved were selected, and the LP in PB, BALF and LNPF were measured and compared.At the same time,the relationship between main respiratory symptoms and LP was preliminarily analyzed.

\section{Methods}

This was a prospective analysis from May 2020 to June 2021. This study was conducted in accordance with 1964 Helsinki Declaration and its later amendments or comparable ethical standards and was approved by the ethics committee of the Beijing Chad-Yang Hospital, Capital Medical University. Written informed consent were obtained from patients or parents/ guardians.

\section{Patients}

16 consecutive patients with sarcoidosis (Radiographic stage $I I, \geq 18$ years old), admitted to Beijing Chao-Yang Hospital,Capital Medical University from May 2020 to June 2021, with enlarged mediastinal/hilar lymph nodes ( $\geq 1 \mathrm{~cm}$ in short axis) on CT of the chest and underwent EBUS-TBNA, were enrolled in the study. The sarcoidosis were diagnosed following diagnostic criteria: (1) compatible clinical and/or radiological findings; (2) histological evidence of non-caseating granulomas; and (3) exclusion of other diseases with a similar histological or clinical profile ${ }^{[5]}$. Patients with any of the following were excluded: pregnancy,malignant tumor,a pathologically confirmed non-neoplastic diagnosis other than sarcoidosis, unclear diagnosis or absence of specific explanation for mediastinal adenopathy, autoimmune disease, use of immunosuppressant or immuno-enhancer 2 weeks before admission, serious disease or dysfunction of another organ system,incomplete clinical data and loss to follow-up.

The medical date,which included demographic information,clinical symptoms, imaging findings,bronchoscopic findings,laboratory findings,complications, and outcomes of the patients during hospitalization, were collected and analyzed. According to whether there were respiratory symptoms (cough and/or chest tightness,chest pain), patients were divided into symptomatic group and asymptomatic group.

\section{EBUS-TBNA procedure}

EBUS procedures were performed by operators proficient in the technique. The convex probe EBUS scope (BF-UC 260FW; Olympus Medical Systems, Japan) with a 7.5 MHz transducer and a compatible 
endoscopic ultrasound scanner (EVIS EUS EUME2;Olympus Medical Systems,Japan)and dedicated fine needles (NA-201SX-4021,21 gauge, Olympus,Japan) were used.The International Association for the Study of Lung Cancer map was followed for categorization of lymph node stations ${ }^{[6]}$. The procedure was performed in bronchoscopy room under propofol and fentanyl vein anesthesia. EBUS-TBNA was performed to assess enlarged mediastinal/hilar lymphnodes.Pulse rate, respiratory rate,oxygen saturation by pulse oximetry and blood pressure were monitored throughout the procedure.

we obtained a total of at least six passes( four from one lymph node,two from another one, or six from one lymph node in case only one enlarged nodes are accessible).TBNA samples were pushed out from the puncture needle into a sterile collection bottle containing $20 \mathrm{ml}$ normal saline and the needle was rinsed with the normal saline into the bottle. The solid tissues were Picked out with a needle and fixed in $10 \%$ buffered formalin solution and used for routine pathological examination and acid-fast staining. The remaining $20 \mathrm{ml}$ liquid component was LNPF,5ml for flow cytometry, $5 \mathrm{ml}$ for routine cytology and $5 \mathrm{ml}$ for Xpert MTB/RIF assay.

\section{BAL procedure}

BAL was performed using flexible bronchofiberscopy (Olympus, Tokyo, Japan) immediately after the EBUS-TBNA procedure. Four to five fractions of $20 \mathrm{ml}$ sterile saline were instilled into the most extensively involved pulmonary segment, as determined by chest CT scans. If pulmonary CT abnormalities are only nodules found in subpleural or interlobar fissure, BAL was performed in the middle lobe of the right lung.The BALF was gently retrieved with suction and kept in sterile containers. The BALF were used for microbiological and cytological examinations in addition to the determination of the differential cell count and flow cytometry analysis.

\section{TBLB and EBB procedure}

The decision to perform an endobronchial biopsy (EBB) and transbronchial lung biopsy (TBLB) was left to the discretion of the bronchoscopist. In general, EBB was performed if there were endobronchial abnormalities and routinely in patients with clinical suspicion of sarcoidosis. TBLB was performed if there were any parenchymal abnormalities on CT scans. The EBB and TBLB specimens were subjected to histopathological examination and acid-fast staining.

\section{Flow cytometry of LNPF,BALF and PB}

BALF or LNPF was strained through metal net and centrifuged,and the supernatant was removed. The cell pellet was resuspended in phosphate buffered saline and centrifuged, and the supernatant was removed.The cell pellet was used for flow cytometry analysis using a flow cytometer(BeckmanCoulter DXFlex, Beckman Coulter Commercial Enterprise Co, CA,USA) with monoclonal anti-CD3, anti-CD4, antiCD8, CD16, CD19 and CD56 antibodies from the same manufacturer. After the PB was treated according to the standard process specified by the manufacturer, flow cytometry analysis is carried out according to the same method.

\section{Tuberculin skin test (TST)}


TST was carried out with 5 tuberculin units purified protein derivative (PPD, Beijing Sanroad Biological Products CO,LTD,Beijing,China) using the Mantoux method. Indurations were recorded at $48-72 \mathrm{~h}$; a subject was positive for TST if the induration diameter was $\geq 10 \mathrm{~mm}$.

\section{Detection of Mycobacterium tuberculosis DNA and rifampicin resistance gene}

The detection of Mycobacterium tuberculosis DNA and rifampicin resistance gene in LNPF was completed by genexpert MTB/RIF analysis platform (GeneXpert ${ }^{\circledR}$ DX System R2,GX$\mathrm{XVI}$, Cepheid,CA,USA).LNPF were concentrated by centrifugation and processed by a standardized technique in which $2 \mathrm{ml}$ sample reagent is added to untreated sample. The closed sample container is manually agitated at room temperature and then $2 \mathrm{~mL}$ of the sample is transferred to the test cartridge. Cartridges are then inserted into the test platform for GeneXpert MTB/RIF assay.After an automated cycle lasting about two hours, a print out of results is obtained.The semi-quantitative levels of Xpert results were negative, very low, low, medium, or high.

\section{Histological examination}

Routine pathological examination were performed on specimens from TBNA, TBLB, EBB and superficial lymph nodes.Sarcoidosis granulomas are most often nonnecrotic. Typical granulomatous features include the presence of well-formed, concentrically arranged layers of immune cells, most prominent being the central core of macrophage aggregates and multinucleated giant cells. An outer layer of loosely organized lymphocytes.Acid fast staining were performed on specimens from TBNA.

\section{Statistical analysis}

Categorical variables were described as numbers and percentages (\%) and continuous variables were described as mean, standard deviation (SD), median, and interquartile range (IQR). The Shapiro-Wilk test was used to verify normality. Differences among LNPF,BALF and PB were assessed by a two-sample ttest for normally distributed continuous variables, the Mann-Whitney $U$ test for non-normally distributed continuous variables.Spearman's rank correlation was used to evaluate correlation of LP among LNPF,BALF and PB,and to evaluate correlation between major respiratory symptoms (cough and/or chest tight,chest pain) and LP in LNPF or BALF. A twotailed p-value less than 0.05 was considered statistically significant.

\section{Results}

A total of 16 patients with sarcoidosis, radiographic stage II, were admitted to this study.11 patients were divided into symptomatic group and 5 into asymptomatic group. EBUS-TBNA were performed and Obtained lymph node tissue samples successfully in all cases and typical granulomatous lesions were found in $87.5 \%$ cases(14/16). Typical granulomatous lesions were found in 2 patients in superficial lymph nodes and EBB specimens. 
87.5\% (14/16) cases completed the pulmonary function examination. 7 (7/14) cases had normal pulmonary function.7 (7/14) cases had abnormal pulmonary function, 4 with mild obstruction,2 with small airway dysfunction, 1 with mild restrictive ventilation dysfunction. No case had diffusion dysfunction. Two patients who did not complete pulmonary function examination were all without respiratory symptoms.

GeneXpert MTB/RIF of LNPF, acid-fast staining of pathological specimens and TST were negative in all cases.

The demographics, clinical manifestation, the method of obtaining pathological tissue, the results of TST, genexpert MTB/RIF and acid-fast staining, and the number of granulomas found of all participants were summarized in Table 1. 
Table 1

The demographics and clinical manifestation of study subjects.

\begin{tabular}{|c|c|c|c|}
\hline Patient Number & 16 & Gender M/F & $6 / 10$ \\
\hline Age,yr(mean,range) & $49.4,35-68$ & Smoker (current/former/never) & $2 / 1 / 13$ \\
\hline Underlying condition or illness & & Main symptoms & \\
\hline Diabetes mellitus & 4 & Respiratory symptoms & $11 / 16$ \\
\hline Hypertension & 4 & Cough & $8 / 16$ \\
\hline Pulmonary function test* & $14 / 16$ & Chest tight & $6 / 16$ \\
\hline Normal Pulmonary function & $7 / 14$ & Chest pain & $1 / 16$ \\
\hline Mild obstruction & $4 / 14$ & Weight loss & $1 / 16$ \\
\hline small airway dysfunction & $2 / 14$ & Fatigue & $1 / 16$ \\
\hline Mild restrictive ventilation dysfunction & $1 / 14$ & $\begin{array}{l}\text { Cases of granuloma/cases } \\
\text { of operation }\end{array}$ & $16 / 16$ \\
\hline Positive TST $(\geq++), n$ & $0 / 16$ & TBNA & $14 / 16$ \\
\hline GeneXpert MTB/RIF, n & $0 / 16$ & TBLB & $5 / 7$ \\
\hline Positive acid-fast staining, $n$ & $0 / 16$ & EBB & $1 / 3$ \\
\hline nonnecrotizing granulomatous, $\mathrm{n}$ & $16 / 16$ & $\begin{array}{l}\text { Supraclavicular lymph } \\
\text { node biopsy }\end{array}$ & $1 / 1$ \\
\hline \multicolumn{4}{|c|}{$\begin{array}{l}\text { Abbreviations: TBNA transbronchial needle aspiration, TBLB transbronchial lung biopsy, EBB } \\
\text { endobronchial biopsy, TST Tuberculin skin test. }\end{array}$} \\
\hline \multicolumn{4}{|c|}{ 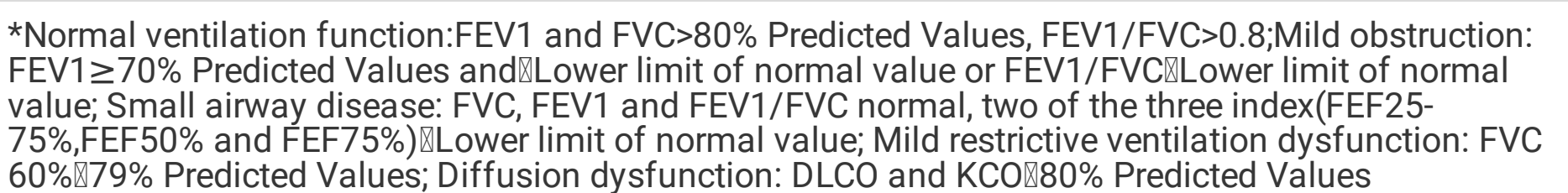 } \\
\hline
\end{tabular}

\section{LP in LNPF,BALF and PB}

We performed LP analysis in LNPF,BALF and PB. The results showed There was no correlation in LP among LNPF, BALF and PB( $P>0.05)$. The results were showed in Table 2 and Figure 1. 
Table 2

The LP in LNPF,BALF and PB[(mean \pm SD) or Median( 25 Percentile,75 Percentile)]

\begin{tabular}{|llll|}
\hline LP & LNPF( $n=16)$ & BALF $(n=16)$ & PB $(n=14)$ \\
\hline LC $(\%)$ & $33.8 \pm 15.9$ & $26.2 \pm 10.1$ & $21.1 \pm 5.5$ \\
\hline TC (\%) & $65.4 \pm 13.8$ & $86.8 \pm 11.6$ & $68.3 \pm 10.3$ \\
\hline CD4(\%) & $46(42.4,58.9)$ & $65.6 \pm 14.6$ & $45.6 \pm 8.1$ \\
\hline CD8(\%) & $10.8 \pm 3.1$ & $12.9 \pm 7.9$ & $20.3 \pm 4.9$ \\
\hline CD4/CD8 & $5.0 \pm 1.7$ & $6.7 \pm 4.0$ & $2.4 \pm 0.8$ \\
\hline BC(\%) & $16.6 \pm 4.9$ & $0.4(0.2,1.0)$ & $9.0 \pm 3.7$ \\
\hline NK(\%) & $1.9(0.7,2.7)$ & $1.1(0.5,2.4)$ & $9.3(7.2,18.7)$ \\
\hline NKT(\%) & $7.4 \pm 5.9$ & $4.2 \pm 2.0$ & $3.0 \pm 1.0$ \\
\hline $\begin{array}{l}\text { Abbreviations: LP: Lymphocyte profile; LNPF: lymph node puncture fluid; BALF: bronchoalveolar } \\
\text { lavage fluid; PB: Peripheral blood; LC\%: Iymphocyte/Tot cells; TC\%:T cell(CD3+ CD19-)/LC, CD4\%: } \\
\text { CD3+CD4+CD8- T cell/LC; CD8\%: CD3+CD4-CD8+ T cell/LC; BC\%: CD3-CD19+ cell/LC; NK\%:CD3- } \\
\text { CD16+ cell/LC; NKT\%: CD3+CD16+ CD56+ T cell/LK. }\end{array}$ & \\
\hline
\end{tabular}

Our results showed that compared with the LP in BAL, in LNPF the proportion of lymphocytes and B cells increased, the proportion of T lymphocytes, CD4 T cells, CD4/CD8 ratio and NKT cells decreased, and there was no difference in the proportion of CD8 T cells and NK cells.

Compared with the LP in PB, the results showed in LNPF the proportion of lymphocytes, B cells, CD4/CD8 ratio and NKT cells increased, the proportion of CD8 T cells and NK cells decreased, and there was no difference in the proportion of T and CD4 T cells.

Compared with the LP in PB, the results showed in BALF the proportion of T lymphocytes, CD4 T cells and CD4/CD8 ratio increased, the proportion of CD8 T cells, B cells and NK cells decreased, and there was no difference in the proportion of lymphocytes and NKT cells.

\section{Correlation between respiratory symptoms and LP in LNPF,BALF and PB}

The results showed that there was no correlation between LP in LNPF and in BALF in all patients and in symptomatic patients. There was no correlation between LP in LNPF and respiratory symptoms. There were correlation between T cells, CD4, CD8 T cells, CD4/CD8 ratio in BALF and respiratory symptoms and there were no correlation between lymphocytes, B cells, NK cells ,NKT cells in BALF and respiratory symptoms. There were correlation between CD8 T cells in PB and respiratory symptoms $(r=-0.628, P=0.016)$. The results of correlation between $L P$ in $B A L F$ and respiratory symptoms are shown in Table 3. 
Table 3

The correlation between respiratory symptoms and LP in BALF

\begin{tabular}{|c|c|c|c|c|c|c|c|c|}
\hline & LC \% & $\mathrm{T} \mathrm{C} \%$ & CD4\% & CD8\% & CD4/CD8 & B C $\%$ & NK\% & NKT\% \\
\hline$r$ & -0.073 & 0.659 & 0.600 & -0.804 & 0.804 & -0.397 & -0.439 & -0.132 \\
\hline $\mathrm{p}$ & 0.788 & 0.006 & 0.014 & 0.000 & 0.000 & 0.128 & 0.089 & 0.626 \\
\hline
\end{tabular}

\section{LP in LNPF and BALF in symptomatic group and asymptomatic group}

The subgroup analysis showed that There was no significant difference in LP in LNPF between symptomatic group and asymptomatic group. There were significant differences in T cells, CD4, CD8 T cells and CD4/CD8 ratio in BALF between the symptomatic group and asymptomatic group. There were no significant differences in lymphocytes, B cells, NK cells and NKT cells in BALF between the symptomatic group and the asymptomatic group. The results were showed in Table 4.

Table 4

The LP in LNPF and BALF in symptomatic group and asymptomatic group.

\begin{tabular}{|lllllll|}
\hline LP & LNPF & \multicolumn{5}{c|}{ BALF } \\
\cline { 2 - 6 } & Sym(11) & Asym(5) & $\mathbf{p}$ & Sym(11) & Asym(5) & p \\
\hline LC(\%) & $39.0 \pm 14.7$ & $25.1 \pm 16.4$ & 0.145 & $25.8 \pm 10.9$ & $27.2 \pm 8.9$ & 0.796 \\
\hline T LC(\%) & $65.3 \pm 14.7$ & $65.7 \pm 13.3$ & 0.959 & $91.7(85.8,98.5)$ & $80.52 \pm 3.2$ & 0.009 \\
\hline CD4(\%) & $45.6(42.2,58.4)$ & $52.1 \pm 13.7$ & 0.661 & $73.2(68.4,78.8)$ & $55.2 \pm 9.7$ & 0.019 \\
\hline CD8(\%) & $10.6 \pm 3.5$ & $11.3 \pm 2.3$ & 0.672 & $8.4 \pm 3.3$ & $22.9 \pm 5.5$ & 0.000 \\
\hline CD4/CD8 & $5.1 \pm 1.8$ & $4.8 \pm 1.7$ & 0.913 & $8.9 \pm 2.6$ & $1.9 \pm 1.1$ & 0.000 \\
\hline B C(\%) & $16.2 \pm 9.9$ & $17.6 \pm 19.2$ & 0.854 & $0.3(0.1,0.8)$ & $0.5(0.35,7.3)$ & 0.145 \\
\hline NK(\%) & $2.2(0.8,2.8)$ & $1.4 \pm 0.9$ & 0.320 & $1.0(0.4,2.3)$ & $4.4 \pm 4.5$ & 0.09 \\
\hline $\begin{array}{l}\text { NKT(\%) } \\
\text { 7.1 }\end{array}$ & $8.1 \pm 6.3$ & 0.441 & $3.9 \pm 1.7$ & $4.8 \pm 2.6$ & 0.427 \\
\hline $\begin{array}{l}\text { Abbreviations: LP: } \text { Lymphocyte profile; LNPF: lymph node puncture fluid; BALF: bronchoalveolar } \\
\text { lavage fluid; Sym: Symptomatic group; Asym: asymptomatic group. LC\%: Iymphocyte/Tot cells; TC\% }\end{array}$ \\
$\begin{array}{l}\text { T cell(CD3+ CD19-)/LC, CD4\%: CD3+CD4+CD8- T cell/LC;CD8\%:CD3+CD4-CD8+T cell/LC;BC\%: CD3- } \\
\text { CD19+ cell/LC; NK\%: CD3-CD16+ cell/LC;NKT\%:CD3+CD16+ CD56+ T cell/LC. }\end{array}$ \\
\hline
\end{tabular}




\section{Discussion}

With the use of EBUS-TBNA, lymph node biopsy by EBUS-TBNA has become a routine examination for mediastinal/hilar lymphadenopathy in the field of respiratory medicine ${ }^{[7]}$. Our study also confirmed that EBUS-TBNA was successfully performed in all patients, and pathological specimens were successfully obtained. 87.5\% (14 / 16) of the patients pathologically confirmed non necrotizing granuloma, which was consistent with $\mathrm{Oda}(84.8 \%)^{[8]}$. Only a few patients had transient fever after operation, indicating that EBUS-TBNA is a safe and efficient examination method for sarcoidosis patients with mediastinal/hilar lymphadenopathy.

In our study, we completed TBNA with a $21 \mathrm{G}$ puncture needle with a larger diameter to ensure sufficient pathological and cell samples. Solid tissue samples were used for pathological examination, and cell samples mixed with a fixed volume of normal saline were used for flow cytometry. Our study not only does not increase the number of puncture, but also takes into account pathology and flow cytometry at the same time. The LNPF and BALF specimens were treated and detected by the same method in our study.Compared with the detection of LP in LNPF by immunohistochemistry and in BALF by flow cytometry and with different solvents to treat LNPF and BALF samples in other studies,our method was simple and more suitable for routine clinical operation. The quality control was reliable, and the results were more comparable and reproducible.Meanwhile, the negative results of acid fast stain performed in tissues specimens and genexpert MTB/RIF performed in LNPF, combined with TST negative were used to help eliminate tuberculosis more accurately.

About $86 \%$ 97\% of patients with sarcoidosis have hilar lymph node enlargement and about $31 \% \sim 48 \%$ have lung infiltration.It was reported that $30 \% \sim 53 \%$ of sarcoidosis patients may have respiratory symptoms, $27 \%$ 53\% may have cough, and $18 \%$ 51\% may have dyspnea, $9 \% \sim 23 \%$ may have chest pain $^{[9,10]}$.Our study found that $68.8 \%$ of the patients in this group had respiratory symptoms, which was higher than the above reported results. It may be related to the fact that we only selected stage II patients, while most stage I patients had no respiratory symptoms. we analyzed the relationship between respiratory symptoms and LP in LNPF,BALF and PB. The results showed that the LP in LNPF were not correlated with respiratory symptoms. Only CD8 T cells of LP in PB were negatively correlated with respiratory symptoms. Parts of LP in BALF were affected by respiratory symptoms. The T cells, CD4 T cells and CD4/CD8 ratio in BALF were positively correlated with respiratory symptoms and were higher in symptomatic group than those in asymptomatic group. The CD8 T cells in BALF were negatively correlated with respiratory symptoms and were lower in symptomatic group than those in asymptomatic group. Total lymphocytes, B cells, NK cells and NKT cells in BALF were not correlated to respiratory symptoms, and there was no significant difference between symptomatic group and asymptomatic group.

Similar to our findings, Danila et al found a large increase in the percentage of CD4 T cells and CD4/CD8 ratio and a decrease in the percentage of CD8 T cells in BALF of sarcoid patients with Löfgren's syndrome compared with asymptomatic patients ${ }^{[11]}$. Drent et al. identified that patients with erythema nodosum 
and/or arthralgia and hilar lymphadenopathy have the highest CD4/CD8 ratio compared with other sarcoid patients ${ }^{[12]}$. However, different from our study, the above studies focused on the symptoms of Löfgren's syndrome, No attention was paid to respiratory symptoms, nor limited to stage 2 patients.

Our results showed no correlation among LNPF, BALF and PB in LP. Some studies also involved the correlation of LP between LNPF and BALF, but most of them focused on the CD4/CD8 ratio, and the results were different.A few studies reported that CD4/CD8 ratios in the lymph nodes and BALF were significantly correlated ${ }^{[8,13]}$. Sory $\mathrm{J}$ Ruiz reported There was poor concordance between CD4/CD8 ratios in BALF and lymph node tissue ${ }^{[14]}$. However, These studies did not exclude the interference of staging and respiratory symptoms, and different stages determine whether the lung parenchyma is involved. Respiratory symptoms also reflect the bronchopulmonary involvement, while the results of BALF reflect the bronchoalveolar lesions.

T lymphocytes especially CD4 T cells play an important role in the development of granulomas and has helped to distinguish sarcoidosis from other disease ${ }^{[15]}$.There are many studies on lymphocytes, $\mathrm{T}$ cells and CD4 T cells in BALF in sarcoidosis, but there are few studies on LNPF.Our study showed that the proportion of LP between LNPF and BALF is significantly different. Our study showed,the LP in LNPF compared with those in BALF,the proportion of lymphocytes and B cells increased, the proportion of $T$ cells, CD4 T cells, CD4/CD8 ratio and NKT cells decreased, and there was no difference in the proportion of CD8 $\mathrm{T}$ cells and NK cells.

Our study confirmed that CD8 T cells in PB were significantly higher than those in LNPF and BALF, and there was no significant difference in CD8 T cells between LNPF and BALF.Contrary to CD4 T cells, CD8 T cells in PB and BALF were negatively correlated with respiratory symptoms, and the asymptomatic group was higher than the symptomatic group.In line with our results,Parasa $\mathrm{VR}^{[16]}$ reported that different from CD4 T cells,compared with healthy control groups,the number and activity of CD8 T cells in sarcoidosis were increased mainly in PB rather than local granuloma tissue.

Measurement of the CD4/CD8 ratio has been used to differentiate sarcoidosis from other diseases since the $1980 \mathrm{~s}^{[17]}$.In the clinic, a CD4/CD 8 ratio $>3.5$ in BALF is used as a criterion to strengthen the diagnosis of sarcoidosis ${ }^{[17]}$.Increased CD4/CD8 ratio was observed only in some sarcoidosis patients, with a sensitivity of between $54 \%$ and $80 \%$ and a specificity of between $59 \%$ and $80 \%[18,19,20]$.Tanriverdi reported that BALF CD4/CD8 ratio were significantly higher in sarcoidosis than other diffuse parenchimal lung diseases.Best cut off value of CD4/CD8 was 1.34 with sensitivity and specificity $76.4 \%, 79.4 \%$ respectively. The cut off values of CD4/CD8 of $>3.5$ and $>2.5$ had specificity $95.9 \%$ and $95.3 \%$, respectively and sensitivity $52 \%, 41 \%$, respectively ${ }^{[21]}$.

Our results showed that the proportion of CD4 T cells in LNPF was lower than that in BALF.There was no difference in the proportion of CD8 T cells between LNPF and BALF.The LNPF and BALF CD4/CD8 ratio were higher than that in PB and the BALF CD4/CD8 ratio were higher than that in LNPF.In our study the BALF CD4/CD8 ratio are $₫ 3.5$ in $68.8 \%$ (11/16) patients. Combined with clinical respiratory symptoms and 
mucosal changes under bronchoscopy, it is found that patients below 3.5 have no respiratory symptoms such as cough, chest tightness and normal bronchial mucosa. Patients higher than 3.5 had respiratory symptoms, bronchial mucosal congestion and edema, and even granular nodules. The results also confirmed that the BALF CD4/CD8 ratio was related to respiratory symptoms, and the BALF CD4/CD8 ratio in symptomatic group was higher than that in asymptomatic group.The reason may be that respiratory symptoms and abnormality of bronchial mucosa reflect that the lavage site is the lesion site involved by sarcoidosis, while no respiratory symptoms and abnormality of bronchial mucosa indicate that the lavage site is not affected by sarcoidosis.

Our results showed that the LNPF CD4/CD8 ratio was slightly lower than BALF.There was no significant difference between symptomatic group and asymptomatic group. The LNPF CD4/CD8 ratio were $₫ 3.5$ in $81.3 \%(13 / 16)$ cases. If taking CD4/CD8 ratio $\varangle 3.0$ as the cut-off value, $93.8 \%(15 / 16)$ patients fell above the cut-off value in LNPF, and $68.8 \%(11 / 16)$ patients fell above the cut-off value in BALF

There were a few studies on LNPF CD4/CD8 ratio and the detecting methods were also different, and there were significant differences in the results. In line with our results, Oda reported that the CD4/CD8 ratio in BALF and lymph nodes was 6.1 and 3.6, respectively.The LNPF CD4/CD8 ratio was lower than BALF.In this study the immunohistochemical method was used for LNPF CD4/CD8 ratio determination and flow cytometry method for BALF CD4/CD8 ratio determination ${ }^{[8]} \cdot P$ Darlington also reported in pulmonary sarcoidosis the LNPF CD4/ CD8 ratio was significantly lower compared with BALF. All 15 patients had a BALF CD4/CD8 ratio >3.5 and 6(6/15) patients had a LNPF CD4/CD8 ratio >3.5. In this study aspirates were obtained with a very fine $25 \mathrm{G}$ needle under EUS-FNA via the oesophagus and the sample processing of aspirates was not described in detail ${ }^{[22]}$.Sory J Ruiz reported the CD4/CD8 ratio was higher in BALF than that in LNPF(4.9 vs 3.5,mean) in sarcoidosis. In this study 1 to 2 additional dedicated passes performed specifically for flow cytometry and the sample processing of aspirates was not described in detail ${ }^{[14]}$. Different from the above results,Ken Akao reported in sarcoidosis the CD4/CD8 ratio was significantly higher in lymph nodes than in the BAL fluid $(7.55 \pm 3.98$ vs $4.91 \pm 3.63, P<0.0002)$, which may be related to the specimen treatment method.In this study, all 2-3 puncture specimens, including solid tissue specimens, were made into cell suspension for flow cytometry and the flow cytometry specimens contained more lymph node components and less blood components ${ }^{[13]}$.

The role of B cells in sarcoidosis has also attracted attention in recent years. Histologically, in addition to a large number of CD4 T cells, there are also B cells in the periphery of granuloma. B cells infiltration can also be seen in the center of some granuloma tissues.Sarcoidosis is often associated with hypergammaglobulinaemia, autoantibody production and circulating immune complexes ${ }^{[23,24,25,26]}$.The case report that rituximab can improve lung function in patients with chronic sarcoidosis also suggests that $B$ cells play a role in the pathogenesis of sarcoidosis ${ }^{[27]}$.Kudryavtsev also found that the frequency of $\mathrm{B}$ cells of sarcoidosis patients was higher than that of healthy controls in $\mathrm{PB}^{[28]}$. $\mathrm{B}$-cell accumulation has been shown in granuloma and pulmonary lesions, and a positive effect of B-cell depletion has been reported $^{[28,29]}$.In our study the proportion of B cells in LNPF was higher than that in BALF and PB.It 
suggested that B cells seem to be involved in several processes in sarcoidosis pathogenesis and could be used to assess disease activity and could possibly be used in the diagnosis of sarcoidosis. However, further research is required.

Natural killer (NK) cells are the core cells of the natural immune system, derived from bone marrow stem cells. They are a group of large granular lymphocytes different from $T$ and B cells, mainly distributed in peripheral blood, liver and spleen.NK cells are part of the first-line defense of the immune system, Lower $\mathrm{NK} \%$ was observed in BALF from sarcoidosis than other interstitial lung diseases ${ }^{[30,31]}$.NK cells in BALF are associated with poor outcome and an advanced radiological stage.An impaired lung function was shown to be associated with a high level of NK cells in the lungs. Furthermore, sarcoidosis patients requiring steroid treatment also had a higher percentage of these cells in BALF ${ }^{[32]}$. Our study found that the proportion of NK cells in LNPF and BALF were significantly lower than that in PB and there was no significant difference in the proportion of NK cells in LNPF and BALF in sarcoidosis patients. The lower proportion of NK cells in LNPF and BALF in our study may be related to the mild condition and slight impairment of pulmonary function in our stage $\otimes$ patients. The proportion of NK cells in LNPF and BALF may help to distinguish the sarcoidosis patients requiring steroid treatment.

NKT cells, a unique subgroup of lymphocytes with features of both T and NK cells, represent a bridge between innate and adaptive immunity.In sarcoidosis, NKT cells have been found at reduced levels in blood and BAL fluid. Regarding NKT cells in granulomas of patients with sarcoidosis, the results are conflicting.Kobayashi $S$ et al.reported an accumulation of NKT cells in granulomatous lesions ${ }^{[3]}$. Mempel $\mathrm{M}$ et al. reported that NKT cells were undetectable in cutaneous sarcoidosis ${ }^{[34]}$. Ho LP et al. reported that the NKT cells were not observed in mediastinal lymph nodes or granulomatous lesions.compared 60 patients with sarcoidosis to healthy control subjects, NKT cells were markedly reduced in both blood and BAL fluid, except in patients with Löfgren syndrome. Because Löfgren syndrome is usually associated with resolving disease, this observation suggests that the loss of NKT cells may allow for persistence of sarcoidosis ${ }^{[35]}$. Our study found that NK T cells in LNPF were significantly higher than those in BALF and PB. NK T cells in LNPF were not correlated with those in BALF and $\mathrm{PB}$, nor with respiratory symptoms. There was no significant difference in NK T cells between BALF

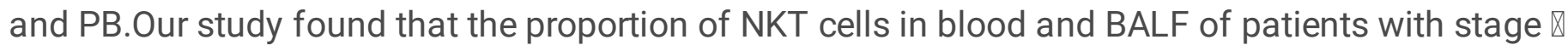
sarcoidosis was lower, while that in LNPF was higher, which may be related to the fact that the patients we selected were stage $\triangle$ patients with short on set time, but did not select patients with chronic persistent disease and advanced patients with pulmonary interstitial fibrosis. The relationship between the change of the proportion of NKT cells in LNPF and patient outcome needs to be further studied.

The similar clinical, imaging, and pathologic manifestations of sarcoidosis and tuberculosis are prone to a misdiagnosis which may result in serious consequences as their treatment methods are completely different. The Xpert MTB/RIF assay,a rapid test recommended by WHO,is a cartridge-based, semiautomated, hemi-nested, real time PCR, which permits rapid diagnosis of tuberculosis through detection of the DNA of M. tuberculosis and concurrent identification of the major mutation that confers rifampicin 
resistance ${ }^{[36]}$.ONE study reported the role of Xpert MTB/RIF on lymph node aspirate in differentiating tuberculosis from sarcoidosis. In TB patients with mediastinal lymphadenopathy undergoing EBUSTBNA, the sensitivity, specificity, positive and negative predictive values of Xpert MTB/RIF in the diagnosis of tuberculosis were $49.1 \%, 97.9 \%, 92.9 \%$ and $77.3 \%$, respectively ${ }^{[37]}$. Our study found that the result of Xpert MTB/RIF on LNPF was negative in all patients, which was consistent with TST, pathological acid fast staining and final clinical diagnosis.It was confirmed that Xpert MTB/RIF on LNPF is a rapid and specific method to exclude lymph node tuberculosis.

There were some limitations in our study. First, the sample size was relatively small, leading to the possibility of selection bias. Second, because we focused only on sarcoidosis, the LP in other benign diseases such as other infectious lymphadenitis are unknown. Whether LP can be used to distinguish sarcoidosis from other benign lymphadenopathies may be an interesting clinical issue. Third, this study was performed at a single academic center in China. Although we used the generalized and well-accepted procedures of EBUS-TBNA and flow cytometric analyses, it may be difficult to exclude potential institutional bias. Furthermore, ethnic bias might have a large influence on inflammation during sarcoidosis.

\section{Conclusion}

1. All specimens obtained by EBUS-TBNA are put into normal saline, solid tissue specimens can meet the needs of pathological examination, and liquid cell specimens (LNPF) can meet the needs of LP examination with the same method(flow cytometry)as BALF is used.

2. LP in LNPF are not correlated with BALF and PB.The LP in LNPF were significantly different from those in BALF: the proportion of lymphocytes and B cells in LNPF was higher than that in BALF, the proportion of $T$ cells, CD4, NK T cells and CD4/CD8 ratio were lower than that in BALF, and the proportion of CD8 T cells and NK cells was not significantly different from that in BALF.

3. LP in LNPF are not correlated with respiratory symptoms, mainly reflect the involvement of lymph nodes, which may be more conducive to the diagnosis and differential diagnosis of sarcoidosis patients with lymph nodes involved.

4. $T$ cells, CD4 T cells and CD4/CD8 ratio in BALF were positively correlated with respiratory symptoms, while CD8 in BALF and PB was negatively correlated with respiratory symptoms. These indexes may more accurately reflect the pulmonary involvement of sarcoidosis.

\section{Abbreviations}

LP, lymphocyte profile; BALF, bronchoalveolar lavage fluid; LNPF, lymph node puncture fluid; PB, peripheral blood; EBUS-TBNA, endobronchial ultrasound-guided transbronchial needle aspiration; EUS-FNA, endoscopic ultrasound-guided fine-needle aspiration; TBLB, transbronchial lung biopsy; EBB, endobronchial biopsy; TST, tuberculin skin test; CT, computed tomography; 


\section{Declarations}

Compliance with ethical standards.

Ethics approval and consent to participate: This prospective study involving human participants was approved by the ethics committees of the Beijing Chao-Yang Hospital, Capital Medical University and was in accordance with the 1964 Helsinki Declaration and its later amendments or comparable ethical standards. Written informed consent were obtained from patients or parents/ guardians.

Consent for publicationपWritten informed consent were obtained from patients or parents/ guardians.

Availability of data and materials: All data generated or analysed during this study are included in this published article.The anonymous data set is available from the corresponding author.

Competing interests[The authors declare that they have no conflicts of interest.

Authors' contributions $\square$ All Authors read and approved the manuscript in the "Authors' Contributions" section. Conception and design: SM, JW, LMZ; Administrative support: JW, LMZ; Provision of study materials or patients:SM, NC, JW, LMZ; Collection and assembly of data: SM, NC; Data analysis: SM, NC, JW, LMZ; Manuscript writing:SM, JW, LMZ; Final approval of manuscript: All authors. LMZ and JW contributed equally to this article and shared corresponding authorship.

Funding This work was supported by the Construction funds of Shijingshan key support specialty (related to epidemic prevention and control) to Dr Jing Wang.

Acknowledgments: We would like to thank the following doctors for taking part in the diagnosis and treatment of patients with sarcoidosis: Ling Wang, Jun Zhang from the Department of Pulmonary and Critical Care Medicine, Beijing Chao-Yang Hospital, Capital Medical University, Beijing, People's Republic China.

Affiliations: ${ }^{1}$ Department of Pulmonary and Critical Care Medicine, Beijing Chao-Yang Hospital, Capital Medical University, Beijing, People's Republic China; ${ }^{2}$ Beijing Institute of Respiratory Medicine, Beijing, People's Republic China.

\section{References}

1. Drent M, Mansour K, Linssen C. Bronchoalveolar lavage in sarcoidosis. Semin Respir Crit Care Med. 2007 Oct;28(5):486-95.

2. Statement on sarcoidosis. Am J Respir Crit Care Med. 1999 Aug;160(2):736-55.

3. Meyer KC, Soergel P. Variation of bronchoalveolar lymphocyte phenotypes with age in the physiologically normal human lung. Thorax. 1999 Aug;54(8):697-700. 
4. Dhooria S, Sehgal IS, Aggarwal AN, Agarwal R. Convex-probe Endobronchial Ultrasound: A Decade of Progress. Indian J Chest Dis Allied Sci. 2016 Jan-Mar;58(1):21-35.

5. Crouser ED, Maier LA, Wilson KC, et al.Diagnosis and Detection of Sarcoidosis. An Official American Thoracic Society Clinical Practice Guideline. Am J Respir Crit Care Med. 2020 Apr 15;201(8):e26-e51.

6. Rusch VW, Asamura $H$, Watanabe $H$, et al.The IASLC lung cancer staging project: a proposal for a new international lymph node map in the forthcoming seventh edition of the TNM classification for lung cancer. J Thorac Oncol. 2009 May;4(5):568-77.

7. Trisolini R, Lazzari Agli L, Tinelli C, et al. Endobronchial ultrasound-guided transbronchial needle aspiration for diagnosis of sarcoidosis in clinically unselected study populations. Respirology. 2015 Feb;20(2):226-34.

8. Oda $\mathrm{K}$, Ishimoto $\mathrm{H}$, Yatera $\mathrm{K}$, et al. Relationship between the ratios of CD4/CD8 T-lymphocytes in the bronchoalveolar lavage fluid and lymph nodes in patients with sarcoidosis. Respir Investig. 2014 May;52(3):179-83.

9. Mañá J, Rubio-Rivas M, Villalba N,et al. Multidisciplinary approach and long-term follow-up in a series of 640 consecutive patients with sarcoidosis: Cohort study of a 40-year clinical experience at a tertiary referral center in Barcelona, Spain. Medicine (Baltimore). 2017 Jul;96(29):e7595.

10. Sinha A, Lee KK, Rafferty GF,et al. Predictors of objective cough frequency in pulmonary sarcoidosis. Eur Respir J. 2016 May;47(5):1461-71.

11. Danila E, Jurgauskiene L, Norkuniene J,et al. BAL fluid cells in newly diagnosed pulmonary sarcoidosis with different clinical activity. Ups J Med Sci. 2009;114(1):26-31.

12. Drent $M$, van Velzen-Blad $H$, Diamant $M$,et al. Relationship between presentation of sarcoidosis and $T$ lymphocyte profile. A study in bronchoalveolar lavage fluid. Chest. 1993 Sep;104(3):795-800.

13. Akao K, Minezawa T, Yamamoto N,et al. Flow cytometric analysis of lymphocyte profiles in mediastinal lymphadenopathy of sarcoidosis. PLoS One. 2018 Nov 19;13(11):e0206972.

14. Ruiz SJ, Zhang Y, Mukhopadhyay S. CD4/CD8 Ratio in Mediastinal Lymph Nodes Involved by Sarcoidosis: Analysis of Flow Cytometry Data Obtained by Endobronchial Ultrasound-guided Transbronchial Needle Aspiration. J Bronchology Interv Pulmonol. 2016 Oct;23(4):288-297.

15. Kantrow SP, Meyer KC, Kidd P, Raghu G. The CD4/CD8 ratio in BAL fluid is highly variable in sarcoidosis. Eur Respir J. 1997 Dec;10(12):2716-21.

16. Parasa VR, Forsslund $H$, Enger T,et al. Enhanced $C D 8^{+}$cytolytic $T$ cell responses in the peripheral circulation of patients with sarcoidosis and non-Löfgren's disease. Respir Med. 2018 May;138S:S38S44.

17. Costabel U. CD4/CD8 ratios in bronchoalveolar lavage fluid: of value for diagnosing sarcoidosis? Eur Respir J. 1997 Dec;10(12):2699-700.

18. Chopra A, Kalkanis A, Judson MA. Biomarkers in sarcoidosis. Expert Rev Clin Immunol. 2016 Nov;12(11):1191-1208. 
19. Tanriverdi H, Erboy F, Altinsoy B,et al. Bronchoalveolar Lavage Fluid Characteristics of Patients With Sarcoidosis and Nonsarcoidosis Interstitial Lung Diseases: Ten-Year Experience of a Single Center in Turkey. Iran Red Crescent Med J. 2015 Oct 28;17(10):e31103.

20. Hyldgaard C, Kaae S, Riddervold M,et al. Value of s-ACE, BAL lymphocytosis, and CD4+/CD8+ and CD103+CD4+/CD4+ T-cell ratios in diagnosis of sarcoidosis. Eur Respir J. 2012 Apr;39(4):1037-9.

21. Tanriverdi H, Uygur F, Örnek T,et al. Comparison of the diagnostic value of different lymphocyte subpopulations in bronchoalveolar lavage fluid in patients with biopsy proven sarcoidosis. Sarcoidosis Vasc Diffuse Lung Dis. 2016 Jan 15;32(4):305-12.

22. Darlington $\mathrm{P}$, Haugom-OIsen $\mathrm{H}$, von Sivers $\mathrm{K}$,et al. T-cell phenotypes in bronchoalveolar lavage fluid, blood and lymph nodes in pulmonary sarcoidosis-indication for an airborne antigen as the triggering factor in sarcoidosis. J Intern Med. 2012 Nov;272(5):465-71.

23. Dall'Aglio PP, Pesci A, Bertorelli G, Brianti E, Scarpa S. Study of immune complexes in bronchoalveolar lavage fluids. Respiration. 1988;54 Suppl 1:36-41.

24. Hunninghake GW, Crystal RG. Mechanisms of hypergammaglobulinemia in pulmonary sarcoidosis. Site of increased antibody production and role of T lymphocytes. J Clin Invest. 1981 Jan;67(1):8692.

25. Weinberg I, Vasiliev L, Gotsman I. Anti-dsDNA antibodies in sarcoidosis. Semin Arthritis Rheum. 2000 Apr;29(5):328-31.

26. Daniele RP, McMillan LJ, Dauber JH,et al. Immune complexes in sarcoidosis: a correlation with activity and duration of disease. Chest. 1978 Sep;74(3):261-4.

27. Sweiss NJ, Lower EE, Mirsaeidi M,et al. Rituximab in the treatment of refractory pulmonary sarcoidosis. Eur Respir J. 2014 May;43(5):1525-8.

28. Kudryavtsev I, Serebriakova M, Starshinova A,et al. Imbalance in B cell and T Follicular Helper Cell Subsets in Pulmonary Sarcoidosis. Sci Rep. 2020 Jan 23;10(1):1059.

29. Cinetto F, Compagno N, Scarpa R, Malipiero G, Agostini C. Rituximab in refractory sarcoidosis: a single centre experience. Clin Mol Allergy. 2015 Sep 1;13(1):19.

30. Bergantini L, Cameli P, d'Alessandro $M$, et al.NK and NKT-like cells in granulomatous and fibrotic lung diseases. Clin Exp Med. 2019 Nov;19(4):487-494.

31. Sokhatska O, Padrão E, Sousa-Pinto B,et al. NK and NKT cells in the diagnosis of diffuse lung diseases presenting with a lymphocytic alveolitis. BMC Pulm Med. 2019 Feb 13;19(1):39.

32. Tutor-Ureta P, Citores MJ, Castejón R,et al. Prognostic value of neutrophils and NK cells in bronchoalveolar lavage of sarcoidosis. Cytometry B Clin Cytom. 2006 Nov 15;70(6):416-22.

33. Kobayashi S, Kaneko Y, Seino K,et al. Impaired IFN-gamma production of Valpha24 NKT cells in nonremitting sarcoidosis. Int Immunol. 2004 Feb;16(2):215-22.

34. Mempel M, Flageul B, Suarez F,et al. Comparison of the T cell patterns in leprous and cutaneous sarcoid granulomas. Presence of Valpha24-invariant natural killer T cells in T-cell-reactive leprosy 
together with a highly biased T cell receptor Valpha repertoire. Am J Pathol. 2000 Aug;157(2):50923.

35. Ho LP, Urban BC, Thickett DR,et al. Deficiency of a subset of T-cells with immunoregulatory properties in sarcoidosis. Lancet. 2005 Mar 19-25;365(9464): 1062-72.

36. Lawn SD, Mwaba P, Bates M,et al. Advances in tuberculosis diagnostics: the Xpert MTB/RIF assay and future prospects for a point-of-care test. Lancet Infect Dis. 2013 Apr;13(4):349-61.

37. Dhooria S, Gupta N, Bal A,et al. Role of Xpert MTB/RIF in differentiating tuberculosis from sarcoidosis in patients with mediastinal lymphadenopathy undergoing EBUS-TBNA: a study of 147 patients. Sarcoidosis Vasc Diffuse Lung Dis. 2016 Oct 7;33(3):258-266.

\section{Figures}




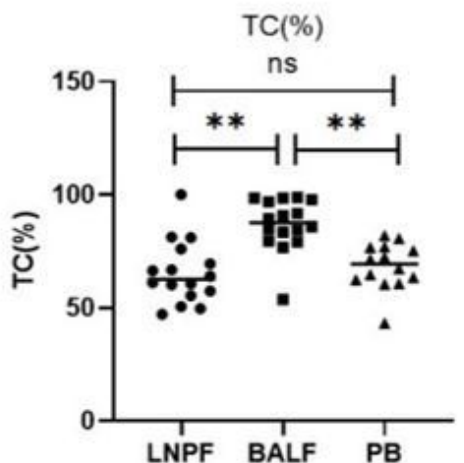

(b)

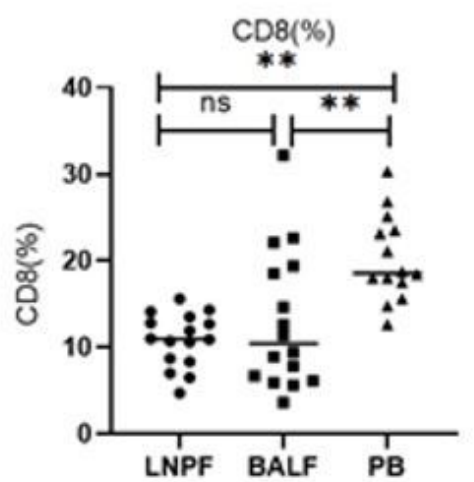

(d)

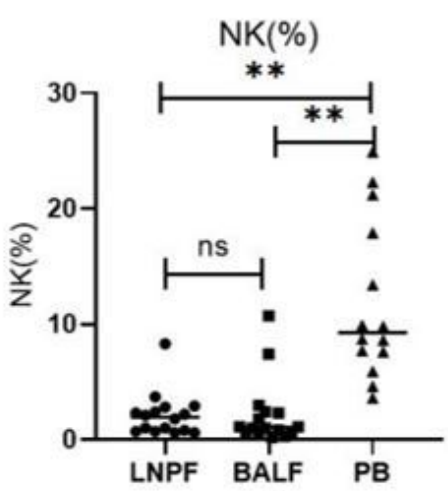

(g)

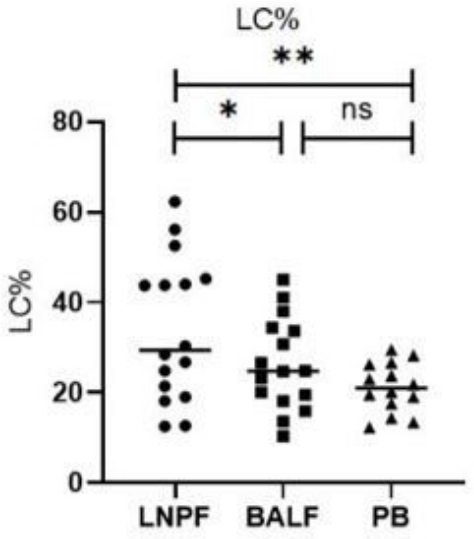

(a)

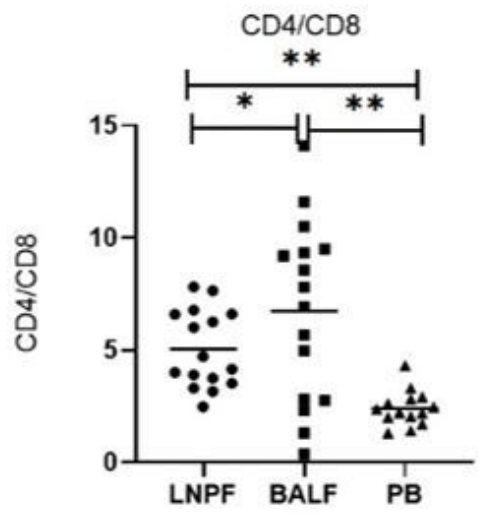

(e)

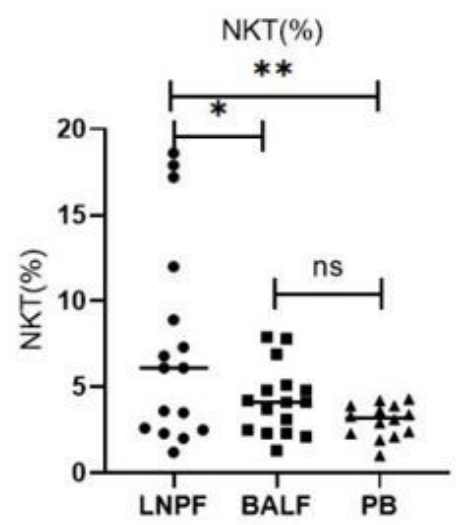

(h)

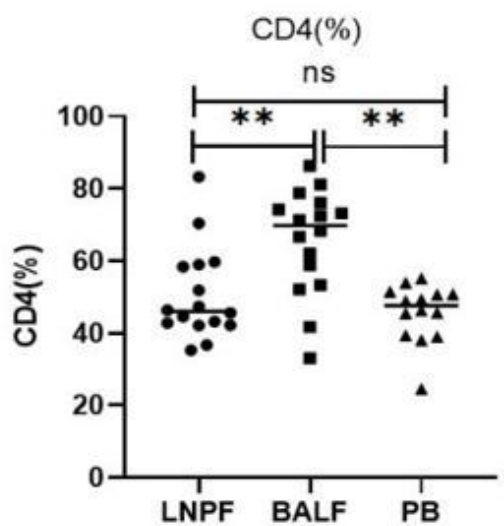

(c)

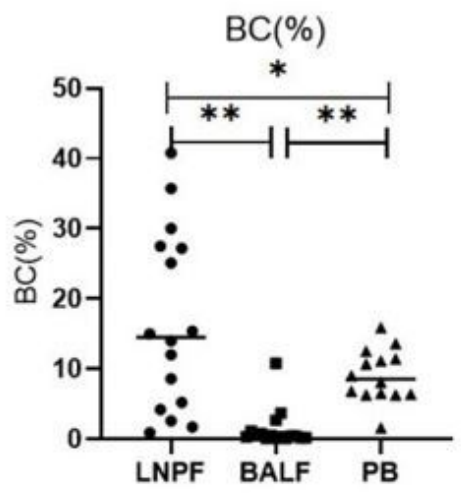

(f)

Figure 1

The LP in LNPF,BALF and PB *:Pख0.05;**:Pख0.01;ns:Pख0.05;@:LNPF; $: B A L F ; \mathbf{A}: P B$

Abbreviations:LP:Lymphocyte profile;LNPF:lymph node puncture fluid;BALF: bronchoalveolar lavage fluid; PB:Peripheral blood;LC\%: lymphocyte/Tot cell;TC\%:T cells(CD3+ CD19-)/LC, CD4\%: CD3+CD4+CD8- T cell/LC; CD8\%: CD3+CD4- CD8+ T cell/LC;BC\%: CD3-CD19+ cell/LC; NK\%:CD3-CD16+ cell/LC; NKT\%: CD3+CD16+ CD56+ T cell/LK. 